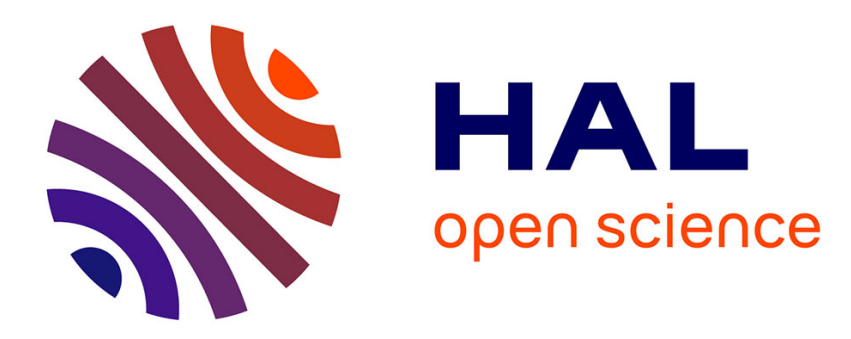

\title{
Esquisse d'une étude théorique de rentabilité des machines forestières
}

\author{
R. Brunet
}

\section{To cite this version:}

R. Brunet. Esquisse d'une étude théorique de rentabilité des machines forestières. Revue forestière française, 1961, 5, pp.297-310. 10.4267/2042/24371 . hal-03386300

\section{HAL Id: hal-03386300 https://hal.science/hal-03386300}

Submitted on 19 Oct 2021

HAL is a multi-disciplinary open access archive for the deposit and dissemination of scientific research documents, whether they are published or not. The documents may come from teaching and research institutions in France or abroad, or from public or private research centers.
L'archive ouverte pluridisciplinaire HAL, est destinée au dépôt et à la diffusion de documents scientifiques de niveau recherche, publiés ou non, émanant des établissements d'enseignement et de recherche français ou étrangers, des laboratoires publics ou privés. 


\section{REVUE FORESTIERE FRANÇAISE}

1961

MAI

No 5

Dans ce numéro: R. BRUNET: Esquisse d'une étude théorique de rentabilité des machines forestières. - G. de la Fouchardière: Bois de talus. - R. LEROY: Lignes électriques et fonctionnement des capitaux dans les peupleraies. - M. Cointat : Note sur la constitution des taillis en Champagne humide.

\section{ESQUISSE D'UNE ETUDE THÉORIQUE DE RENTABILITE DES MACHINES FORESTIĒRES}

PAR

\section{R. BRUNET}

Centre Technique du Bois

L'apparition de la machine en forêt, pour n'être pas toute récente, peut cependant, - en France au moins - être qualifiée de timide. Maint chantier forestier ne se signale au passant que par 1' " ouie de la cognée », le crissement de la scie et quelque fumée bleue filtrant à travers les cimes. Quel contraste avec telle " operation ») américaine où l'incessant va et vient des camions, l'activité fébrile des grues et des treuils et la pétarade vindicative des tronçonneuses signalent à la ronde l'installation en ce point d'une véritable antenne industrielle.

Tant pis pour les poètes. En ce siècle, le rendement, l'efficacité, la productivité sont des critères de vie ou de mort, économiquement parlant.

Mais chacun sait aussi que l'on ne mécanise pas au petit bonheur. Aboutissement presque inévitable, la mécanisation exige, pour réussir, que l'on s'astreigne à en étudier les étapes et à en calculer les moyens. Entre une machine, beau jouet convoité, sur la lecture de quelque prospectus, et celle qui sera vraiment l'outil efficace et économique permettant de rester compétitif, il y a souvent un abime.

La présente étude a pour objet d'esquisser une méthode permettant d'escompter la rentabilité d'une mécanisation: donc, de prévoir et non plus de constater le résultat d'une telle opération.

Elle a été volontairement limitée, pour l'instant, à une activité précise: l'exploitation d'un taillis. Elle est, bien entendu, applicable, - moyennant les correctifs nécessaires - à un certain nombre d'autres activités. 
Ainsi définie et cernée, l'entreprise est encore téméraire: on sait les différences considérables que l'on peut constater entre un taillis et un autre. Aussi précisons-nous tout de suite que l'application de cette méthode à /un cas ou à un ensemble de cas particuliers, exige l'adaptation exacte des divers paramètres, et que les conclusions qu'on en tirera peuvent être, pour un tel cas d'espèce, différentes des conclusions générales énoncées ci-après. Cette restriction n'ôte, à nos yeux, aucune valeur à la méthode elle-même.

Le principe de base est simple: la machine remplace l'homme. Une tâche donnée, exécutée moyennant $Z$ francs par une maind'œuvre non mécanisée, doit être accomplie pour $Z$ francs au maximum par la machine et son ou ses conducteurs et ses aides.

Mais on peut envisager une mécanisation plus ou moins poussée: on peut remplacer 4 hommes, par exemple, par 3 hommes et une petite machine, ou par 1 homme et une machine plus importante.

Voici posés les trois éléments entre lesquels la méthode proposée établit des relations:

- la tâche à accomplir (exemple: un nombre de stères/jour);

- l'effectif (réduit) de la main-d'œuvre;

- la " taille ) de la machine à choisir.

Ici, deux remarques s'imposent:

$1^{\circ}$ Nous avons délibérément laissé de côté le point de vue technique pur. De nombreuses machines forestières ont été réalisées ou sont en cours de réalisation. Leur conception peut faire entrer en jeu des principes fort divers. Nous ne les considérerons ici que sur un plan externe, c'est-à-dire qu'elles ne se matérialiseront que:

- par leur rendement;

- par la main-d’œuvre nécessaire pour les actionner et les desservir;

- par leur prix, disons prix d'achat.

$2^{\circ}$ Dans le même ordre d'idées, nous avons négligé des facteurs importants mais mal chiffrables actuellement: celui de la commodité et du confort apportés à l'ouvrier et celui de l'attrait psychologique d'un outil mécanique.

Mais revenons au problème économique posé: comme dans toute activité industrielle, les principales données sont:

-.. le prix d'achat de la matière première (ici le stère sur pied). A - le prix de vente du produit façonné (chargé sur camion) V 
- les frais de fabrication, comprenant essentiellement les salaires dans le processus traditionnel.

Selon les considérations évoquées plus haut, nous verserons dans ce poste le coût d'amortissement et de fonctionnement des machines remplaçant partiellement la main-d'œuvre traditionnelle. Donc: salaires et machines (au stère). ......

- Nous grouperons dans un poste unique les frais généraux (dans lesquels seront inclus les frais de prospection, de réception des produits, etc...) et le bénéfice (ramené au stère). G Enfin, nous poserons une première hypothèse: à savoir que les frais et le bénéfice $G$, dans une exploitation bien gérée, représentent environ $20 \%$ du prix de revient $\mathrm{V}-\mathrm{A}$

$$
\mathrm{G}=\frac{20(\mathrm{~V}-\mathrm{A})}{100}
$$

ou

$$
\mathrm{T}=\frac{80}{100}(\mathrm{~V}-\mathrm{A})
$$

Ces considérations sont valables pour tous les cas où une exploitation est naturellement rentable dans l'hypothèse envisagée.

Or, on sait bien que, dans le cas du taillis exploité par une méthode traditionnelle, les frais de fabrication sont fréquemment trop élevés, même si le prix d'achat $\mathrm{A}$ est réduit à des valeurs très faibles :

$$
\mathrm{T}>\frac{80}{100}(\mathrm{~V}-\mathrm{A})
$$

La mécanisation, dans ce cas, devra résoudre le problème, en réduisant la main-d'œuvre, sans grever, outre mesure, par ses propres frais d'amortissement et de fonctionnement, le poste " frais de fabrication ).

On conçoit qu'un délicat compromis doive être réalisé ici : c'est à le déterminer que s'essaye la méthode proposée.

Illustrons par un diagramme simple les données en présence: (fig. 1).

1) le prix d'achat du stère sur pied $\ldots \ldots \ldots \ldots \ldots \ldots \ldots \ldots$ A

2) les frais généraux et le bénéfice $\ldots \ldots \ldots \ldots \ldots \ldots \ldots$ G

3) les frais de fabrication selon le processus traditionnel (manuel) $T$ 
4) le prix de revient, somme des trois précédents, représenté

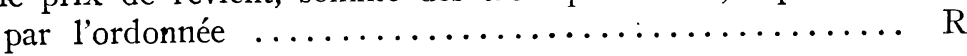

5) le prix de vente $\ldots \ldots \ldots \ldots \ldots \ldots \ldots \ldots \ldots \ldots \ldots$ V inférieur à $\mathrm{R}$ dans le cas général.

Représentons maintenant le remplacement d'une partie des salaires $T$ par des coûts " machine ». Pour cela, traçons sur un axe d'abscisses $\mathrm{x} \mathrm{x}^{\prime}$ : une échelle de 10 intervalles se terminant en $\mathrm{S}$ et joignons $\mathrm{R} \mathrm{S}$.

Par rapport à l'axe $\mathrm{x} \mathrm{x}^{\prime}$, les ordonnées des divers points de la droite R S représentent les salaires versés dans chaque hypothèse (part main-d'œuvre: $90 \%, 80 \%, 70 \%, \ldots$ ).

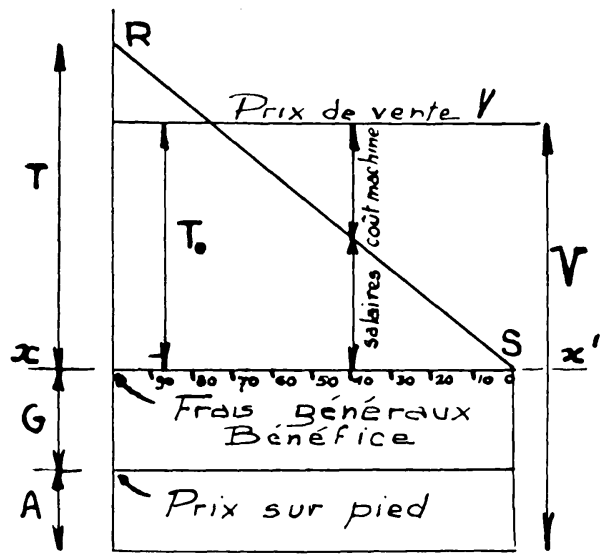

FIg. 1.

De même, les distances de ces points à la droite $\mathrm{V}$ représentent les coûts machine qu'il ne faut pas dépasser pour rester rentable.

Quelques considérations mathématiques élémentaires nous amènent alors aux résultats suivants:

Désignons par $n$ le nombre de stères produit chaque jour par les $\mathrm{N}$ ouvriers du chantier mécanisé

et soit:

$\mathrm{p}$ le coût machine (par stère),

$r$ le pourcentage d'ouvriers conservés dans le chantier mécanisé par rapport à l'effectif du chantier traditionnel,

$\mathrm{n}_{0}$ le nombre de stères exécutés normalement par jour par un ouvrier " manuel »,

$T_{0}$ le montant des frais de fabrication à ne pas dépasser pour que notre exploitation soit rentable $\left(\mathrm{T}_{\mathrm{o}}\right.$ est l'ordonnée de la droite $\left.\mathrm{V}\right)$. 
On obtient une expression de $p$ que nous allons utiliser dans quelques instants:

$$
\mathrm{p}=\mathrm{T}(1-\mathrm{r})-\left(\mathrm{T}-\mathrm{T}_{\mathrm{o}}\right)=\mathrm{T}_{\mathrm{o}}-\mathrm{Tr}
$$

et

$$
\mathrm{N}=\frac{\mathrm{nr}}{\mathrm{n}_{\mathrm{o}}} \quad \text { donc } \mathrm{r}=\frac{\mathrm{Nn}_{\mathrm{o}}}{\mathrm{n}}
$$

d'où

$$
\mathrm{p}=\frac{\mathrm{T}_{\mathrm{o}} \mathrm{n}-\mathrm{TNn}_{\mathrm{o}}}{\mathrm{n}}
$$

Il ne reste plus, maintenant qu'à trouver une expression synthétisant convenablement les caractéristiques des machines appelées à remplacer une partie de la main-d’ouvre.

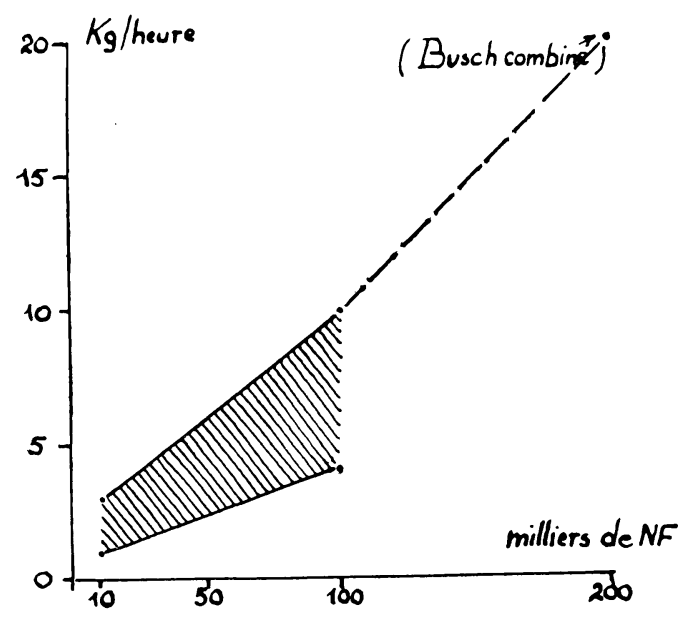

FIG. 2.

Fourchette adoptée pour les consommations de carburant en fonction de la « taille 》 de la machine (exprimée par son prix d'achat). Le carburant a été décompté à $0,27 \mathrm{NF}$ le litre (fuel détaxé). On a ajouté $30 \%$ à ce prix pour les lubrifiants.

Ceci ne va pas, bien entendu, sans obliger à poser certaines hypothèses: sinon, le problème ne pourrait pas être traité dans son cas général. 
Les divers paramètres à considérer ont été fixés aux valeurs suivantes qu'on peut considérer comme des moyennes:

Durée d'amortissement des machines $\ldots . . . . . . . . . .$. .

Intérêt des capitaux investis.. Réparations $\ldots \ldots \ldots \ldots \ldots . .$. Energie et lubrifiant $\ldots \ldots \ldots$. Nombre de jours de fonctionnement réel par an .........
5 ans

$8 \%$

50 à $80 \%$ de la valeur d'achat 2,5 à $30 \mathrm{NF}$ (voir fig. 2)

120

Si nous désignons par $\mathrm{F}$ les frais de base annuels: annuité d'amortissement, annuité d'intérêts, annuité de réparations (supposées réparties sur la durée d'amortissement) nisé,

par $\mathrm{n}$ le nombre de stères produits par jour par le chantier méca-

par $C$ la dépense journalière de carburant et de lubrifiant

$\mathrm{p}$, le prix de revient par stère de la machine (sans son conducteur) s'exprime évidemment par la relation

qu'on peut écrire:

$$
\mathrm{p}=\frac{\mathrm{F}}{120 \mathrm{n}}+\frac{\mathrm{C}}{\mathrm{n}}
$$

$$
\mathrm{n}=\frac{\mathrm{F}+120 \mathrm{C}}{120 \mathrm{p}}
$$

L'expression $\mathrm{F}+120 \mathrm{C}$ est bien représentative de la " taille " de la machine, c'est son coût annuel. Le tableau I donne les valeurs de $\mathrm{F}+120 \mathrm{C}$ pour 10 machines de diverses tailles, selon les conventions ci-dessus.

Il n'est plus maintenant que de remplacer $p$ (expression 2) dans (3) :

$$
\mathrm{n}=\frac{\mathrm{F}+120 \mathrm{C}}{120 \mathrm{~T}_{\mathrm{o}}}+\frac{\mathrm{T}}{\mathrm{T}_{\mathrm{o}}} \mathrm{n}_{\mathrm{o}} \mathrm{N}
$$

Comme nous le voyons, cette ultime expression contient:

- une expression de la taille de la machine $(F+120 \mathrm{C})$

- le nombre de stères produit par le chantier mécanisé (n)

- le nombre (réduit) d'ouvriers desservant le chantier (N)

et des paramètres se référant aux données techniques et économiques de l'exploitation.

Cette expression comporte une représentation simple, puisque linéaire, de la forme donnée à la fig. 3 . 
Une courte explication de ce diagramme permettra d'en démontrer l'utilité.

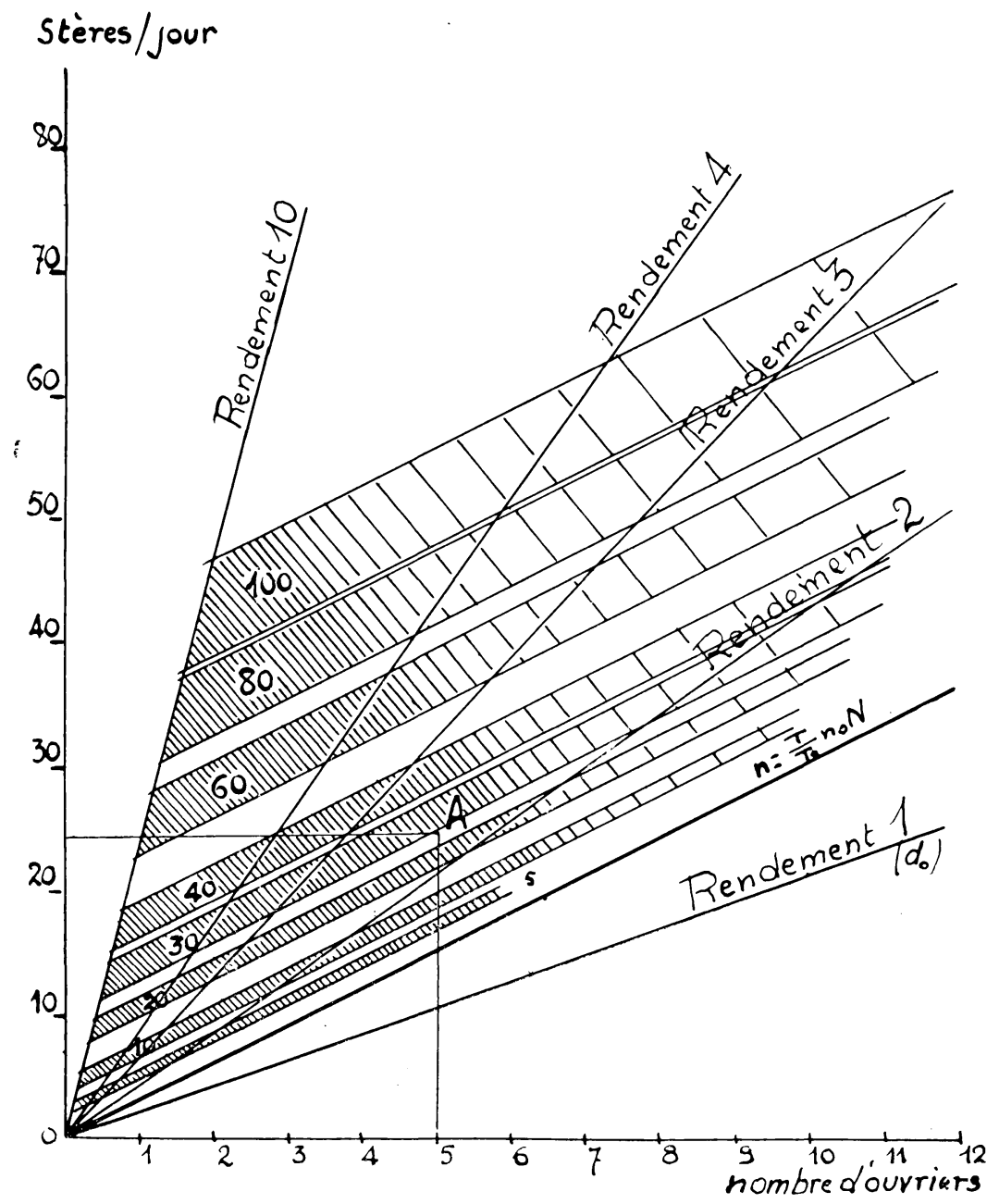

Fig. 3.

La série de plages obliques correspond à des machines de tailles différentes. Elles coupent l'axe des y aux ordonnées correspondant aux diverses valeurs de

$$
\frac{F+120 \mathrm{C}}{120 \mathrm{~T}_{\mathrm{o}}}
$$


Pour la clarté, on trace des plages correspondant à des prix d'achat de machine en chiffres ronds : $10000,20000,30000$ NF ... (voir tableau I).

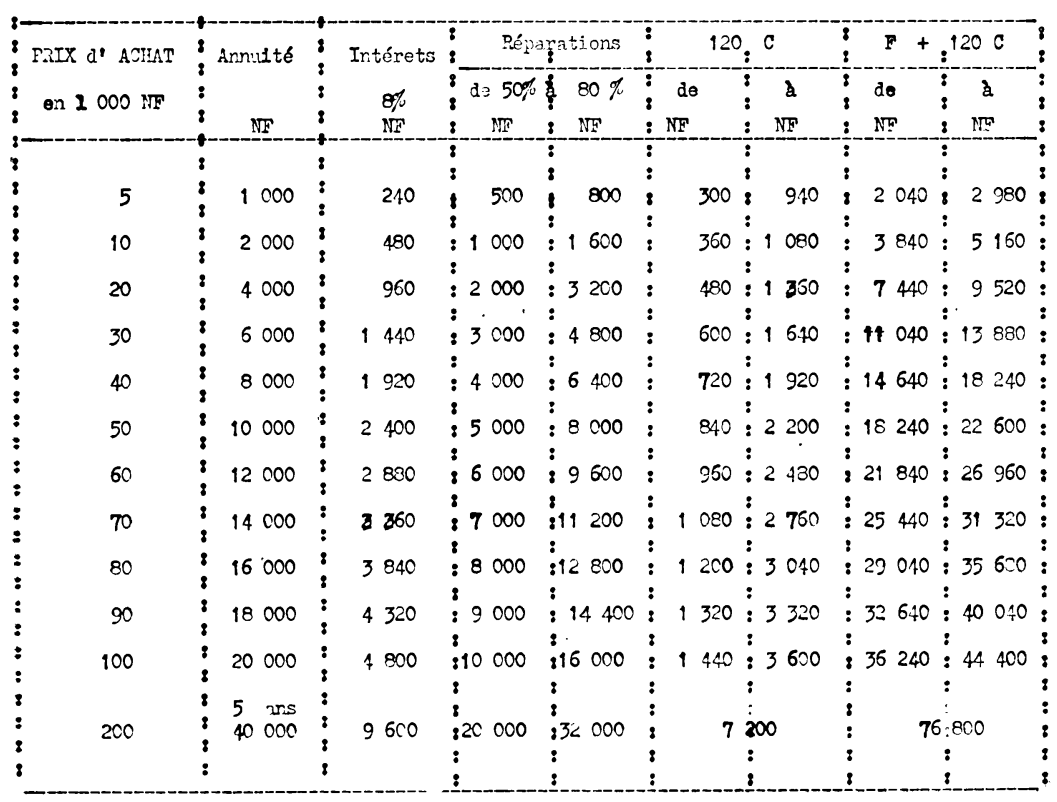

\section{Tableau I}

Leur pente est égale à $\frac{T}{T_{0}} \dot{n_{0}}$, puisqu'elles sont toutes parallèles entre elles et à la droite de base $n=\frac{T}{T_{0}} n_{0} N$. Que penser de cette dernière? C'est celle pour laquelle $(F+120 \mathrm{C})$ est nul, correspondant donc à une mécanisation nulle. Mais elle est théorique, car, sans machine. $\mathrm{N}$ ouvriers ne peuvent produire que $n_{0} N$ stères (droite $\mathrm{d}_{\mathrm{o}}$ ).

En réalité, elle marque qu'avec une main-d'œuvre coûtant $T$ $\left(>T_{0}\right)$, le rendement de cette dernière devrait être de $n_{o} N \times \frac{T}{T_{0}}$, ce qui est évident.

Ceci dit, un tel diagramme permet, connaissant la taille et le rendement d'une machine ou d'un ensemble de machines donné, de déterminer la rentabilité de leur emploi. Il suffit de marquer le 
point A correspondant au rendement journalier (n) et à la maind'œuvre nécessaire $(\mathrm{N})$ et de comparer la position de ce point à la droite correspondant à la taille de la machine.

Si le point A se trouve situé au-dessus de la plage de " taille » ou sur elle, la rentabilité du chantier est assurée. S’il est au-dessous, la machine est trop chère, ou a un rendement insuffisant, ou exige une main-d'œuvre trop nombreuse.

Une notion supplémentaire peut être obtenue en traçant les droites:

$$
\begin{gathered}
\mathrm{n}=2 \mathrm{n}_{\mathrm{o}} \mathrm{N} \\
\mathrm{n}=3 \mathrm{n}_{\mathrm{o}} \mathrm{N} \\
\text { etc... }
\end{gathered}
$$

correspondant à la notion d'augmentation du rendement de la maind'œuvre grâce à la machine: doublement, triplement, etc... Dans l'exemple de la figure 4, le point $\mathrm{A}^{\prime}$ correspond à un chantier mécanisé où le rendement de la main-d'œuvre traditionnelle est doublé.

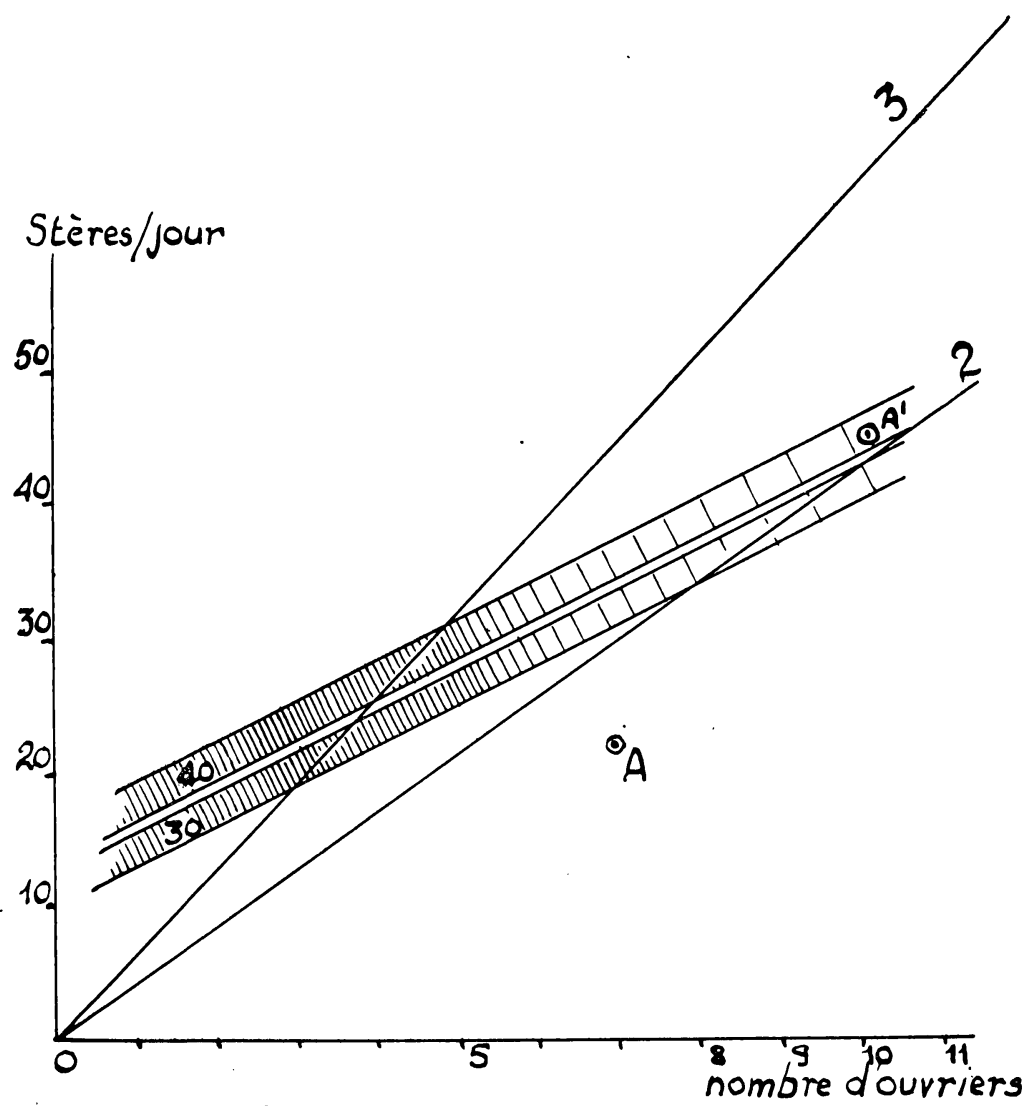

FIg. 4. 
Voici deux exemples d'application de cette méthode:

Premier exemple: Il participe à la fois de l'expérimentation d'un prototype français et de l'étude de son amélioration, à réaliser dans un proche avenir.

Conditions de milieu:

Pour éviter de se placer dans un cas trop particulier, nous prendrons les conditions que l'on rencontre dans beaucoup de taillis français; tout est ramené au stère:

Frais réels de fabrication (bois chargé sur camion: T C C)

$$
\mathrm{T}=12,20 \mathrm{NF}
$$

Détermination de $\mathrm{T}_{0}$

Prix de vente: $16 \mathrm{NF}$ camion départ coupe.

Prix d'achat sur pied: 3 NF (prix choisi volontairement assez élevé, dans l'hypothèse où l'on se place d'assurer une bonne rentabilité de l'ensemble du processus).

Frais généraux - bénéfice: $3,80 \mathrm{NF}$.

$\mathrm{T}_{\mathrm{o}}=16-(3,80+3,00)=9,20 \mathrm{NF}$.

$\mathrm{n}_{\mathrm{o}}$ (rendement journalier de l'ouvrier manuel) - débardage et chargement inclus - est pris égal à 2,3 st.

Caractéristiques de la machine:

C'est une combinée débardeuse - tronçonneuse - chargeuse. Son prix est d'environ $30000 \mathrm{NF}$, auquel il faut ajouter quelques scies d'abattage,

Soit, en tout: $35000 \mathrm{NF}$ environ.

Dans sa version actuelle inachevée, le rendement instantané du chantier correspond à une production théorique de 30 stères/jour, avec 6 ouvriers, dont quelques-uns sont payés plus cher que des bûcherons ordinaires: l'ensemble de la main-d'œuvre, sur le plan du coût, équivaut à 7 bîcherons.

Il est prudent, pour tenir compte des ruptures de cadence inévitables rencontrées dans tous les chantiers et sur les chantiers forestiers en particulier, d'adopter un rendement correspondant à $2 / 3$ environ du rendement théorique.

$$
\frac{30 \times 2}{3}=20 \text { stères, rendement réel escomptable. }
$$

Cela donne le point $A$ sur le diagramme de la figure 4 : machine non rentable.

Dans sa version définitive prévue, cette machine doit pouvoir produire 60 à 70 stères " théoriques ", soit 40 à 46 stères réels par jour avec 9 hommes équivalant à un peu plus de 10 bûcherons. Quelques scies d'abattage supplémentaires étant nécessaires, l'investisse- 
ment calculé s'élève à $38000 \mathrm{NF}$. Le point $\mathrm{A}$ vient en $\mathrm{A}^{\mathbf{1}}$, la rentabilité doit être atteinte et le problème d'une exploitation normalement déficitaire, résolu.

On peut voir en passant que, dans ces conditions, le rendement de la main-d'œuvre est un peu plus que doublé.

Deuxième exemple: On a cherché à placer, - d'après les renseignements obtenus sur cet appareil, - le " Busch Combine » américain, sur le diagramme. Cette machine (fig. 6) qui réalise toutes

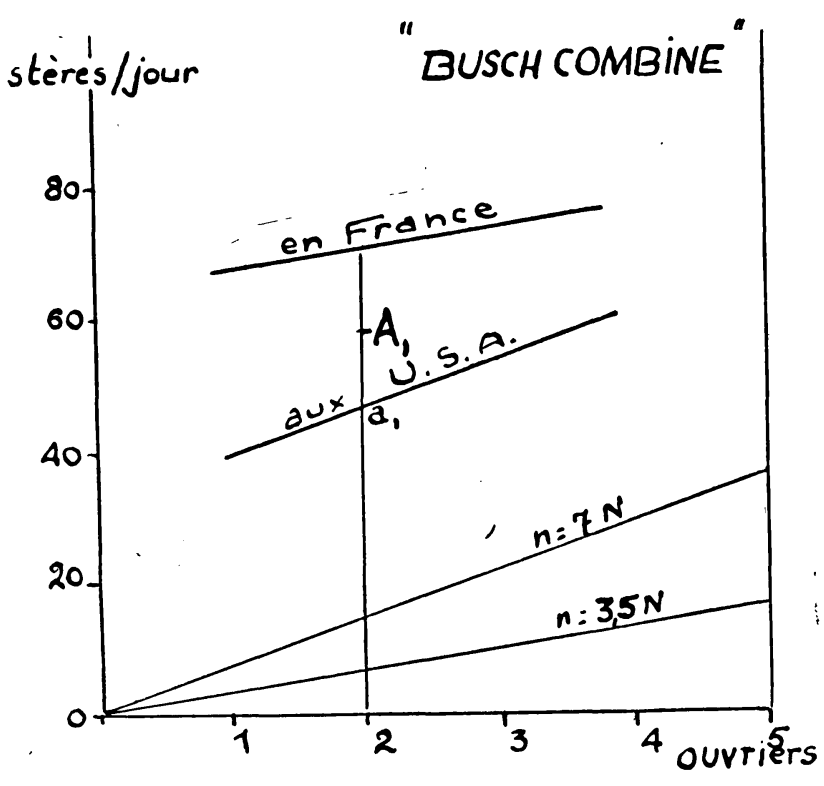

Fig. $\dot{5}$

les phases de l'exploitation avec 2 hommes seulement, jusqu'à l'empilage sur coupe, a un rendement de 58 stères/jour. Elle coûte 38000 dollars; les paramètres correspondants semblent pouvoir être fixés de la manière suivante:

$1^{\circ}$ aux U. S. A., dans des jeunes futaies de pin d'assez fort diamètre, bois tronçonnés en $1,66 \mathrm{~m}$.

$$
\begin{aligned}
& \mathrm{F}+120 \mathrm{C}(1)=19000 \$ \\
& \mathrm{~T}=\mathrm{T}_{\mathrm{o}}=5 \$ \\
& \mathrm{n}_{\mathrm{o}}=7 \text { stères }
\end{aligned}
$$

(1) amortissement en 5 ans, réparations $50 \%$. 
Le point $\mathrm{A}^{1}$ (fig. 5) est nettement au-dessus de la droite de taille: la machine parait parfaitement rentable.

$2^{\circ}$ en France, dans les mêmes conditions forestières:

$$
\begin{aligned}
& \mathrm{F}+120 \mathrm{C}=76800 \mathrm{NF}(1)(2) \\
& \mathrm{T}=\mathrm{T}_{\mathrm{o}}=10 \mathrm{NF} \\
& \mathrm{n}_{\mathrm{o}}=3 \text { stères } 5
\end{aligned}
$$

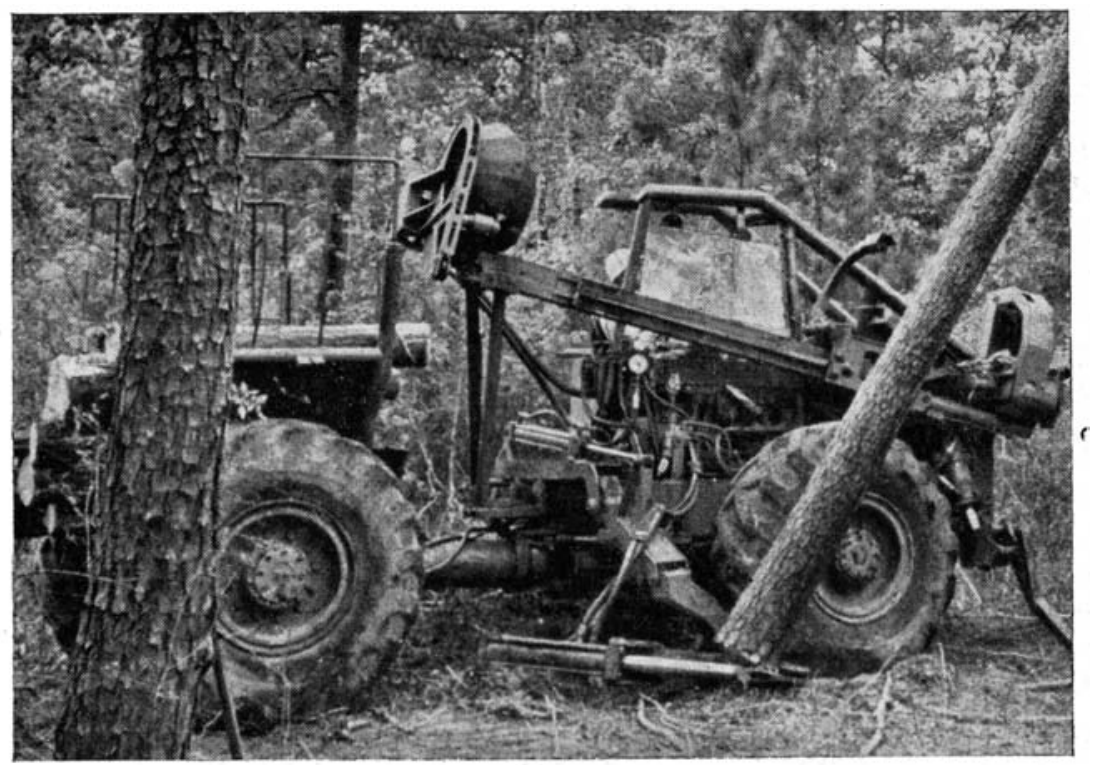

(Cliché international Paper Company.)

Fig. 6.

Busch Combine.

Le point $\mathrm{A}^{1}$ (fig. 5) est, cette fois, au-dessous de la droite de taille: machine non rentable.

Dans un taillis moyen tel que celui choisi dans le premier exemple, l'utilisation du Busch Combine n'est pas pensable.

Cet exemple montre l'influence du niveau des salaires. Ceux-ci entrent au dénominateur $\left(\mathrm{T}_{\mathrm{o}}\right)$ dans le premier terme de l'expression (4), qui corrobore le fait bien connu que, plus les salaires sont élevés, plus la mécanisation est une opération rentable.

(2) prix d'achat supposé: $200000 \mathrm{NF}$. 
Ce deuxième exemple nous a montré une application de la méthode à un chantier naturellement rentable malgré l'emploi de méthodes traditionnelles $\left(T=T_{0}\right)$.

Dans ce cas, on peut aller plus loin et escompter le bénéfice supplémentaire que peut procurer l'emploi d'une telle machine par rapport à une main-d'œuvre manuelle.

En effet, l'ordonnée du point $a^{1}$ (fig. 5) mesure le nombre de stères (43 stères) produits par la machine avec un bénéfice normal, c'est-à-dire identique à celui que l'on aurait obtenu en utilisant 43

$-\frac{7}{7}=6$ bûcherons ordinaires.

La différence entre les ordonnées de $\mathrm{A}^{1}$ et de $\mathrm{a}^{1}$, mesure donc le nombre de stères supplémentaires réalisés " gratuitement 》 par la machine (16 stères).

En stricte théorie, ces stères sont bien gratuits et le bénéfice qu'ils procurent pourrait être décompté tout simplement en adoptant leur prix de vente.

Par exemple, si le bénéfice normal était, disons de $10 \%$ du prix de vente $V$, le nouvearı bénéfice serait ici de:

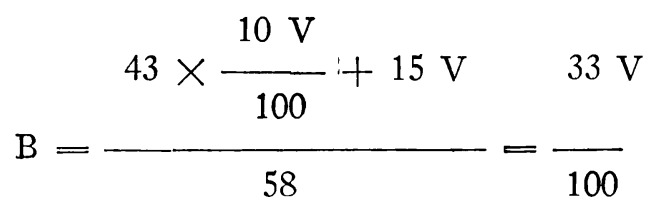

soit $33 \%$ du prix de vente.

Bien ențendu, la réalité n'est peut-être pas aussi belle. V représente, d'ailleurs, ici, un prix empilé sur coupe. Le chargement sur camion nécessite, dans le cas du "Busch Combine " un engin de débardage très important.

Avant de formuler quelques conclusions, quelques remarques s'imposent :

$1^{\circ}$ lorsqu'on emploie plusieurs machines (en les caractérisant par leur prix d'achat), il ne faut pas perdre de vue qu'il ne suffit pas d'additionner leur prix d'achat et se placer sur la droite correspondante du diagramme. Il faut passer par l'intermédiaire du tableau 1, chercher la valeur de $(\mathrm{F}+120 \mathrm{C})$ de chaque machine, les additionner et se rapporter seulement alors au prix global d' " achat » correspondant.

$2^{\circ}$ ne pas perdre de vue que la conduite d'un ou de plusieurs appareils mécanique oblige à envisager des salaires plus élevés pour 
les conducteurs, avec, probablement, un léger effet d'entraînement sur le reste de la main-d'œuvre. Le nombre $\mathrm{N}$ de ("bûcherons ) sera donc déterminé en affectant aux ouvriers hautement spécialisés un chiffre correspondant à cette capacité : $1,4,1,5$, etc..., les bûcherons restant à 1 ou 1,1 , et en additionnant le tout.

$3^{\circ}$ pour les petites machines, inférieures à $5000 \mathrm{NF}$, cette méthode doit être appliquée avec beaucoup de soin. Les divers paramètres doivent être déterminés attentivement pour chaque cas.

$4^{\circ}$ Enfin, il faut bien définir les limites dans lesquelles la méthode trouve son application. Dans l'exemple $\mathrm{N}^{\circ} 1$, nous l'avons considérée pour le processus d'exploitation s'arrêtant au bois chargé sur véhicule routier.

Si on doit l'utiliser pour une série d'opérations plus longue, ou plus courte, il faut choisir avec soin les valeurs atteintes par le bois en début et en fin de série; si c'est impossible, - dans le cas par exemple, où l'on n'étudie que la mécanisation d'une phase de l'exploitation -, il faut déterminer pour quelle part cette phase entre dans le total des coûts de l'exploitation traditionnelle, et n'appliquer la méthode que sur cette part.

Il ne s'agit, comme on le voit, que d'une méthode permettant d'y voir plus clair dans les questions de rentabilité de matériel mécanique appliqué à l'exploitation forestière.

D'ores et déjà, on s'aperçoit vite que la rentabilité d'un chantier manuel normalement déficitaire ou tangent, ne sera obtenue, par mécanisation, que pour une production journalière élevée, et au moins un doublement, mieux un triplement du rendement de la main-d'œuvre traditionnelle. Ce ne sont pas là des conditions réalisables d'un coup de baguette magique: des problèmes de financement et d'organisation se posent obligatoirement.

Enfin, la souplesse d'emploi et la mobilité en tous terrains restent parmi les caractéristiques les plus importantes d'un appareillage mécanique destiné à mobiliser des bois de faibler valeur essaimés sur l'infinité de petits terroirs qui constituent une bonne part du patrimoine forestier national. 